\title{
Reprodução do peixe-rei, Atherinella brasiliensis (Quoy \& Gaimard) (Atheriniformes, Atherinidae), em uma planície de maré adjacente à gamboa do Baguaçu, Baía de Paranaguá, Paraná, Brasil
}

\author{
Luís Fernando Favaro ${ }^{1}$, Sabine de C. G. Lopes ${ }^{2,3} \&$ Henry Louis Spach ${ }^{3}$ \\ ${ }_{1}^{1}$ Departamento de Biologia Celular, Setor de Ciências Biológicas, Universidade Federal do Paraná. Caixa Postal 19031, \\ 81531-990 Curitiba, Paraná, Brasil. E-mail: lufavaro@ufpr.br \\ 2 Programa de Pós-graduação em Genética, Departamento de Genética, Universidade Federal do Paraná. Paraná, Brasil. \\ ${ }^{3}$ Centro de Estudos do Mar, Universidade Federal do Paraná. Avenida Beira Mar, 83255-000 Pontal do Paraná, Paraná, \\ Brasil.
}

\begin{abstract}
Reproduction of silverside,Atherinella brasiliensis (Quoy \& Gaimard) (Atheriniformes, Atherinidae), in a tidal flat fringed by the mangroves of Baguaçu, Paranaguá Bay, Paraná, Brazil. The present study investigated the reproductive biology of silverside, Atherinella brasiliensis (Quoy \& Gaimard, 1825) in a tidal flat of Paranaguá Bay, littoral of Paraná State, Brazil. Fish were captured monthly from November 1998 to October 1999 by the mean of a seine-net. For each individual, total body length, total weight, sex, and gonadal weight were recorded. Gonadal development stages were also defined based on the histological examination of the ovaries and on the visual aspects of the testes. It was defined that the reproductive period of $A$. brasiliensis extends from June to December, based on he analyses of individual gonadosomatic index (GSI) values distribution in relation to the sampling periods, on the monthly variation of the GSI mean values (Curve of Maturation), both for males and females, and on the monthly variation of the relative frequencies of the female maturation stages. During this period, matured, partially spawned and spent females were found in higher frequencies in comparison to the other months. The sizes at first maturation were defined as $7,61 \mathrm{~cm}$ and $6,92 \mathrm{~cm}$ for females and males, respectively.
\end{abstract}

KEY WORDS. Histology, reproductive biology, tidal flat.

No Brasil, poucos estudos ictiológicos realizados em ambientes estuarinos objetivaram a análise da biologia reprodutiva de espécies que habitam as planícies de maré. Estes ambientes apresentam-se expostos ou submersos, pois sofrem a ação das marés, por isso suas variações ambientais são diárias, além daquelas resultantes da sazonalidade.

Em sondagens preliminares realizadas em uma planície de maré no complexo estuarino Baía de Paranaguá, no litoral do Paraná, o peixe-rei Atherinella brasiliensis (Quoy \& Gaimard, 1824) mostrou-se abundante e constante. Segundo Figueiredo \& Menezes (2000), a espécie é encontrada em águas salobras e se distribui da Venezuela ao Rio Grande do Sul.

No Brasil, de acordo com os trabalhos realizados por BARBIERI et al. (1991) na laguna do Marapendi, ANDREATA et al. (1992, 1997) na laguna de Jacarepaguá e na lagoa Rodrigo de Freitas respectivamente, Pessanha et al. (2000) na baía de Sepetiba, a espécie $A$. brasiliensis se mostrou abundante e/ou constante na região estuarina do estado do Rio de Janeiro. Resultados semelhantes foram obtidos por Hostim-SiLva et al. (1995) na Lagoa da Conceição, em Santa Catarina e por Bemvenuti (1987) na região estuarina da Lagoa dos Patos, no Rio Grande do Sul.

Vários estudos de dieta alimentar, aspectos reprodutivo, comportamento reprodutivo, estrutura populacional e recrutamento foram realizados com diferentes espécies de aterinídeos na América do Sul, América do Norte e Europa, dentre estes pode-se citar os estudos de Middaugh et al. (1981), Palmer \& Culley (1983), Conover \& Kynard (1984), Benvenuti (1987), Ortaz \& Guerra (1989), Middaugh \& Hemmer (1992), HostimSilva et al. (1995) e Pessanha \& Araújo (2001).

Pela ausência de um estudo da biologia reprodutiva de A. brasilensis em estuários no estado do Paraná, associado com a abundância e importância ecológica da referida espécie e pela ausência de estudos que abordem a histologia de ovários desta espécie, o presente estudo objetiva caracterizar microscopicamente o desenvolvimento ovariano, determinar a época e o tipo de desova e o comprimento da primeira maturação para fêmeas e machos. 


\section{MATERIAL E MÉTODOS}

Coletas mensais foram realizadas no período de novembro de 1998 a outubro de 1999, em uma planície de maré, adjacente a gamboa do Baguaçu, na Baía de Paranaguá. Com a utilização de uma rede tipo picaré (30,0 m de comprimento X 3,0 m de altura, 2,0 $\mathrm{m}$ de boca, com malhagem de $0,5 \mathrm{~cm}$ entre os nós adjacentes), foram capturados 292 fêmeas e 314 machos, totalizando 606 exemplares da espécie Atherinella brasiliensis.

De cada exemplar foram tomados o comprimento total (cm) e o peso total (g) e posteriormente foram seccionados ventralmente para exposição das gônadas, verificando-se o sexo e avaliando-se macroscopicamente o estádio de desenvolvimento gonadal.

As gônadas foram retiradas, pesadas (g) e algumas de cada coleta foram fixadas em Bouin por 18 horas e posteriormente incluídas em parafinas para confecção de lâminas permanentes, as quais foram coradas com Hematoxilina-Eosina (HE). Os cortes histológicos foram analisados em microscópio de luz e permitiram a caracterização dos estádios de maturação ovariana e a correção da análise macroscópica, quando necessário.

O Índice Gônado-Somático (IGS), definido como a percentagem de participação do peso da gônada em relação ao peso total, foi estimado para cada indivíduo e analisado para sexos separados. Os valores médios mensais foram estimados e a partir da variação temporal, determinou-se a curva de maturação para machos e fêmeas. A determinação do tipo de desova foi baseada na distribuição de Freqüência de ocorrência dos estádios de desenvolvimento ovariano e nas análises microscópicas dos ovários.

A proporção sexual durante o ciclo reprodutivo foi testada pelo método do $\chi^{2}$, com grau de liberdade 1 e 0,05 de significância $\left(\chi^{2}<3,840\right)$, de acordo com VAzzoler (1996).

O tamanho de primeira maturação foi estimado, separadamente para machos e fêmeas, através da curva que relaciona a Freqüência relativa de indivíduos adultos com o ponto médio das classes de comprimento, ajustada pela equação:

$$
\mathrm{fr}=1-\mathrm{e}^{-\mathrm{aLt}}
$$

onde: (fr) freqüência relativa de indivíduos adultos, (e) base do logaritmo neperiano, (a e b) coeficientes estimados pelo método dos mínimos quadrados aplicado na relação linear obtida pela transformação das variáveis envolvidas, $\left(\mathrm{L}_{\mathrm{t}}\right)$ ponto médio das classes de comprimento total.

\section{RESULTADOS E DISCUSSÃO}

\section{Escala de maturidade}

Não se encontram na literatura estudos de reprodução envolvendo atherinídeos, enfocando análises histológicas das gônadas. A utilização destas análises na determinação dos estádios de desenvolvimento ovariano permite um melhor entendimento do processo reprodutivo e, segundo Dias et al. (1998), diminui os erros contidos nas análises macroscópicas.

Para a elaboração da escala de maturidade para as gônadas de fêmeas, faz-se necessário conhecer a ovogênese (desenvolvimento do folículo ovariano), tendo-se adotado no presente trabalho a escala proposta por VAzzoLER (1996).

A escala de maturidade microscópica, confeccionada para fêmeas de $A$. brasiliensis, foi baseada na presença e na quantidade das fases de desenvolvimento do folículo ovariano e está composta por cinco estádios de desenvolvimento. Os estádios B (Maturação) e C (Maduro), estão subdivididos em dois e três sub-estádios respectivamente (Tab. I e Figs 1 e 2 ).

Para A. brasiliensis, os folículos ovarianos a partir da fase III, apresentam um envoltório espessado, incomum aos teleósteos, localizado entre a membrana vitelina e as células foliculares. O envoltório é constituído por vários filamentos dispostos em vários sentidos, sugerindo a função de fixação do folículo ovariano no ambiente. Este resultado detecta a necessidade de futuros estudos abordando este aspecto.

O fato de se investir na microscopia de gônadas femininas, deu-se em decorrência de que as fêmeas, além da produção do gameta feminino, ainda são responsáveis por incorporar nutrientes nos ovócitos para serem utilizados nas primeiras fases do desenvolvimento dos alevinos, tornando o processo de ovogênese mais complexo.

\section{Ciclo reprodutivo}

Através da análise de distribuição dos valores individuais do IGS de machos e fêmeas, bem como das curvas de maturação, constata-se que o desenvolvimento gonadal para ambos os sexos ocorre a partir do mês de junho, com maior desenvolvimento em outubro, seguido de uma diminuição nos valores médios mensais do IGS entre os meses de novembro e janeiro (Figs 3 e 4). A curva de maturação elaborada para a espécie, demonstra um desenvolvimento gonadal sincronizado entre machos e fêmeas (Fig. 4).

Tabela I. Estádios e subdivisões do desenvolvimento ovariano, IGS médio e características microscópicas.

\begin{tabular}{lccl}
\hline \multicolumn{1}{c}{ Estádios } & Subdivisão & IGS médio & Características \\
\hline A (Imaturo) & - & 0,361 & Folículos ovarianos nas fases I e II \\
B (Maturação) & (B1) Maturação Inicial (Fig. 1A) & 0,368 & Presença abundante de folículos ovarianos na fase II e poucos na fase III \\
& (B2) Maturação Final (Fig. 1B) & 2,777 & Folículos ovarianos nas fases II e III (mais abundantes e desenvolvidos) \\
C (Maduro) & (C1) Maduro Inicial (Fig. 1C) & 3,646 & Folículos ovarianos nas fases II, III, IV, V e VI (em início de hialinização) \\
& (C2) Maduro Final (Fig. 2A) & 6,498 & Poucos folículos ovarianos na fase II, e grande quantidade nas fases III, IV, V e VI \\
& (DSVN) Desovando (Fig. 2B) & 5,835 & Folículos ovarianos nas fases II, III, IV, V, VI e folículos vazios \\
SD (Semi desovado) (Fig. 2C) & - & 3,673 & Folículos nas fases II, III, IV e folículos vazios (ausência das fases V e VI) \\
D (Desovado) & - & 1,096 & Folículos ovarianos pré vitelogênicos (fase II) em lamelas desorganizadas \\
\hline
\end{tabular}

Revista Brasileira de Zoologia 20 (3): 501-506, setembro 2003 

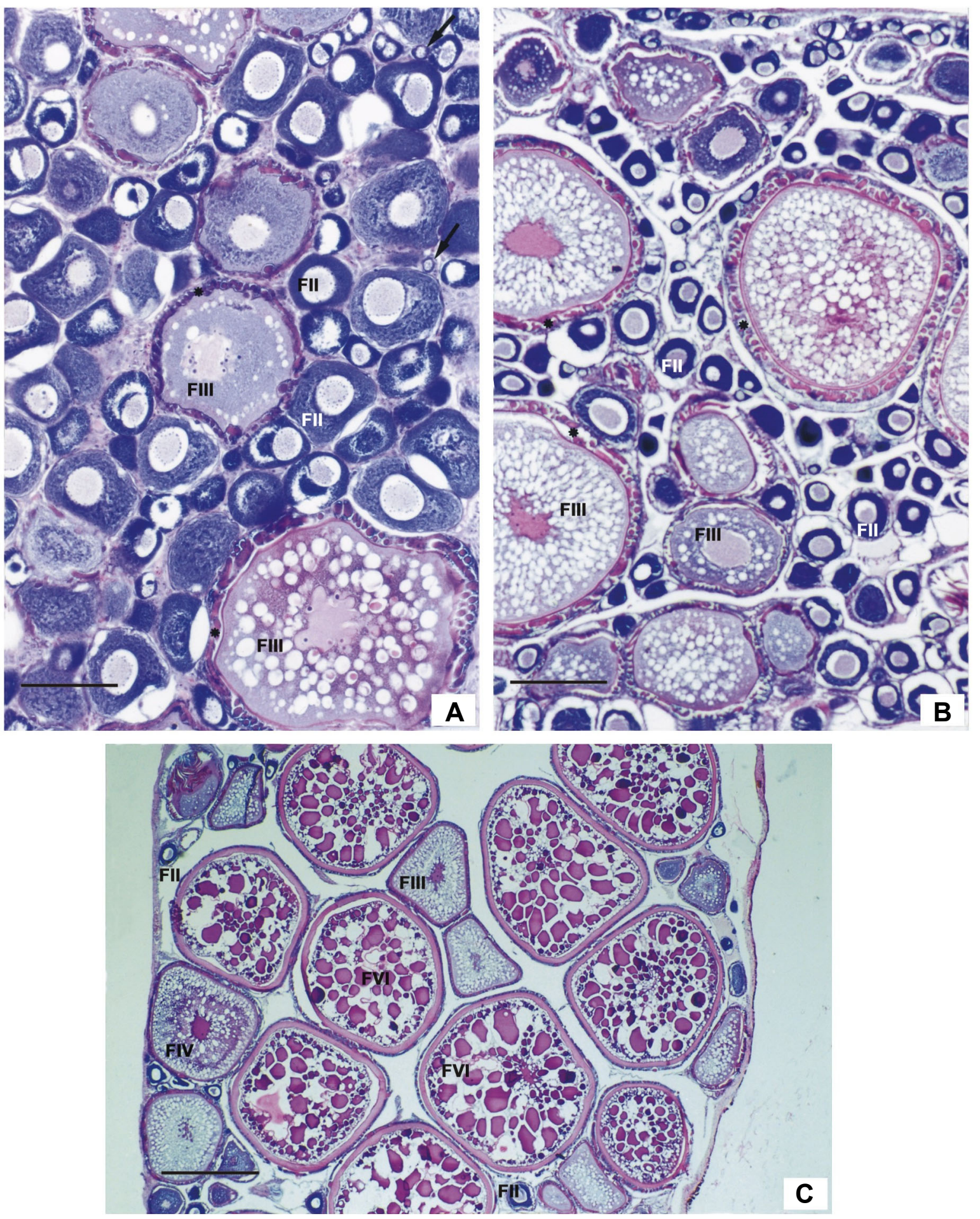

Fig. 1. Cortes histológicos de ovários de A. brasiliensis corados com HE. (A) Ovário em maturação inicial (B1). Folículos ovarianos na fase

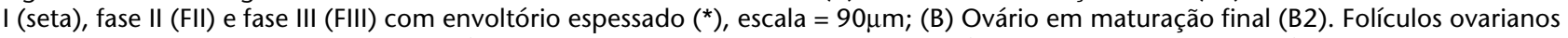
na fase II (FII) e fase III (FIII) com envoltório espessado $\left(^{*}\right)$, escala $=180 \mu \mathrm{m} ;(\mathrm{C})$ Ovário maduro inicial (C1). Folículos ovarianos na fase II (FII), fase III (FIII), fase IV (FIV) e fase VI (FVI) (em início de hialinização), escala $=450 \mu \mathrm{m}$. 

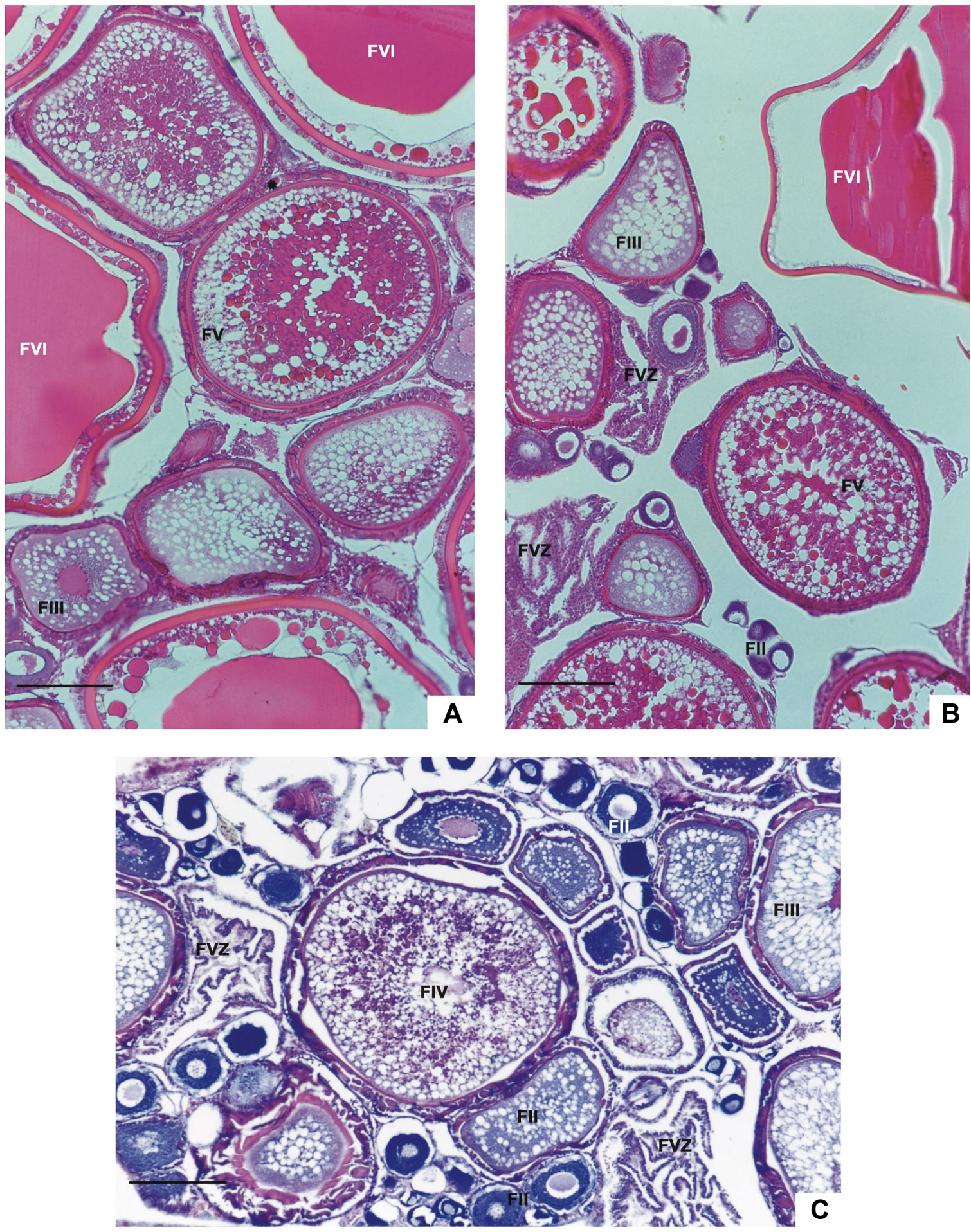

Fig. 2. Cortes histológicos de ovários de A. brasiliensis corados com HE. (A) Ovário maduro final (C2): folículos ovarianos na fase III, fase V (FV) com envoltório espessado (*) e fase VI (FVI) (hialinizado); (B) Ovário desovando (DSVN): folículos ovarianos na fase II (FII), fase III (FIII) e fase V (FV) e folículos vazios (FVZ); (C) Ovário semi desovado (SD): folículos ovarianos na fase II (FII), fase III (FIII), fase IV (FIV) com envoltório espessado $\left(^{*}\right)$ e folículos vazios (FVZ). Escalas $=180 \mu \mathrm{m}$.

Revista Brasileira de Zoologia 20 (3): 501-506, setembro 2003 

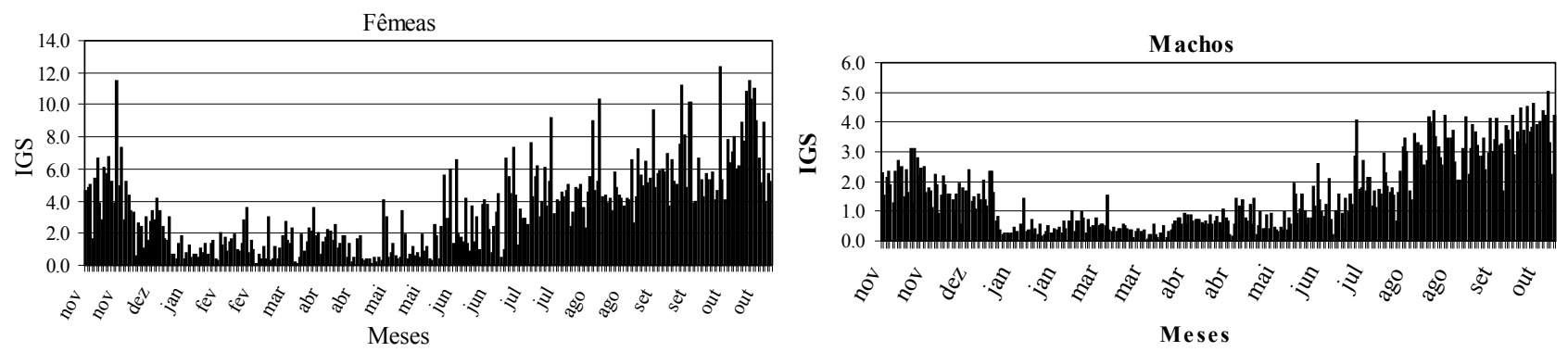

Fig. 3. Distribuição mensal dos valores individuais do IGS de fêmeas e machos no período estudado.

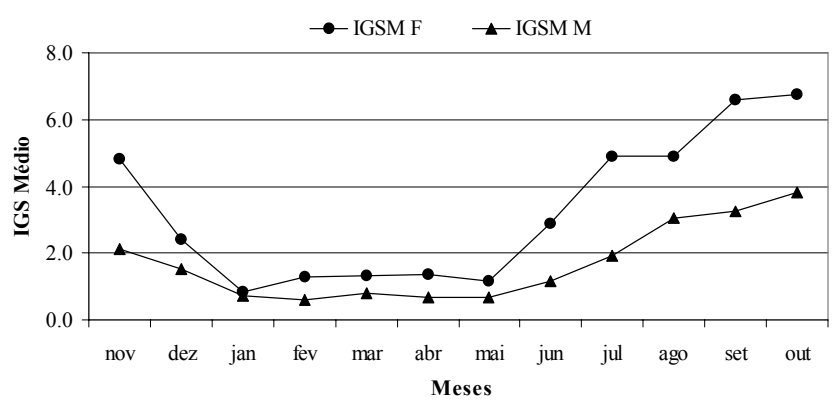

Fig. 4. Curva de maturação para fêmeas e machos de Atherinella brasiliensis: (IGS MF) IGS médio mensal de fêmeas e (IGS MM) IGS médio mensal de machos.

A análise da distribuição dos valores individuais do IGS retrata o desenvolvimento das gônadas de uma referida população de peixes ao longo do ano, considerando cada indivíduo amostrado, permitindo uma maior precisão na análise, considerando-se que valores de IGS dentro de uma variação muito ampla venham a mascarar a média, refletindo este fato nos elevados valores de variância e, conseqüentemente, do coeficiente de variação. Portanto, a utilização dos valores médios do IGS mensal para contribuir na interpretação do ciclo reprodutivo de uma espécie só deve ser empregado quando se tratarem de médias representativas, com real poder de centralização.

Pela análise da distribuição mensal de Freqüência de ocorrência dos estádios de desenvolvimento ovariano e suas subdivisões (Fig. 5), constatou que fêmeas maduras (C1, C2 e/ou DSVN) ocorreram durante todo o ano, enquanto que a Freqüência de fêmeas semi-desovadas foi mais expressiva no período de agosto a fevereiro. Fêmeas apresentando ovários desovados foram registradas nos meses de janeiro a maio, período em que a curva de maturação apresentou os menores valores de IGS médio mensal.

Quando analisados somente os estádios de desenvolvimento ovariano (Fig. 6), agrupando as subdivisões dos estádios B (maturação) e C (maduro), os resultados acima são corroborados. Assim, indivíduos maduros e semi-desovados ocorreram durante todo o ano.

Nas análises realizadas por BEMVENUTI (1987), que utilizou o IGS médio mensal e a distribuição da Freqüência dos diâmetros dos ovócitos, no período de baixos valores de IGS médio, as gônadas foram classificadas no estádio de repouso. No presente estudo, a análise histológica dos ovários e da Freqüência

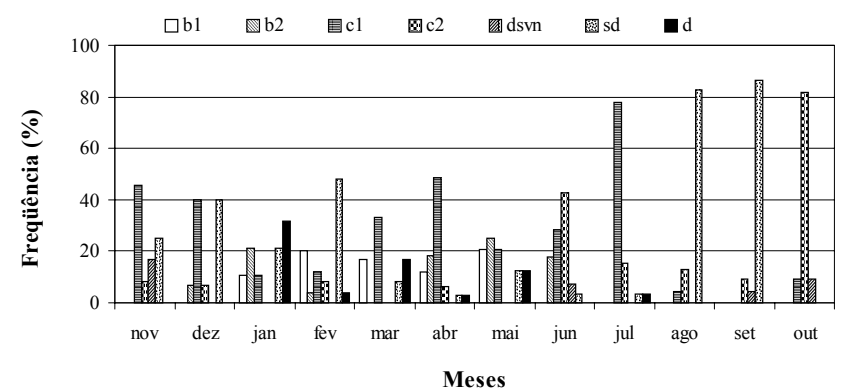

Fig. 5. Freqüência relativa (\%) mensal dos estádios e sub estádios de desenvolvimento ovariano de Atherinella brasiliensis. (B1) Maturação inicial, (B2) maturação final, (C1) maduro inicial, (C2) maduro final, (DSVN) desovando (SD) semi desovado e (D) desovado.

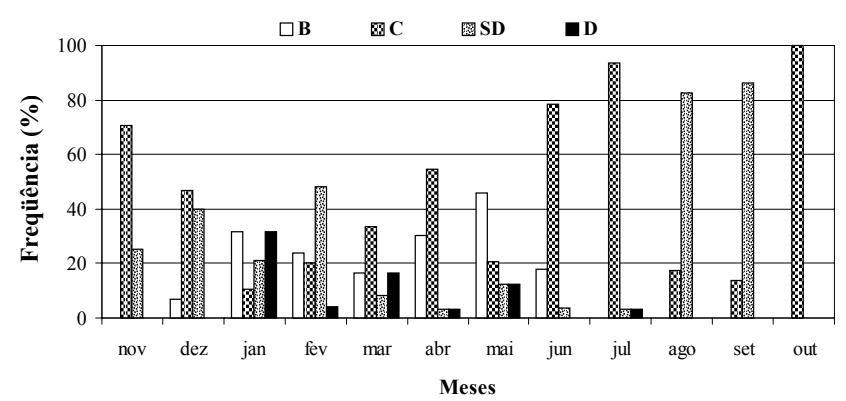

Fig. 6. Freqüência relativa (\%) mensal dos estádios de desenvolvimento ovariano de Atherinella brasiliensis: (B) Maturação, (C) maduro (SD) semi desovado e (D) desovado.

dos estádios ovarianos, efetuadas no período de janeiro a maio, detectou a ocorrência em maior número de fêmeas em maturação (estádio B) e desovadas (estádio D), explicando assim os menores valores do IGS individual e IGS médio mensal.

A análise da distribuição dos valores individuais do IGS, a curva de maturação, a freqüência relativa dos estádios de maturação dos ovários, juntamente com a análise histológica, permitiram verificar que o período reprodutivo ocorre entre os meses de junho a dezembro, corroborado pela alta Freqüência de ocorrência de fêmeas com ovários maduros e semi desovados. A proporção sexual entre machos e fêmeas, durante o período reprodutivo, apresentou valores significativos $\left(\chi^{2}>3,840\right)$

Revista Brasileira de Zoologia 20 (3): 501-506, setembro 2003 

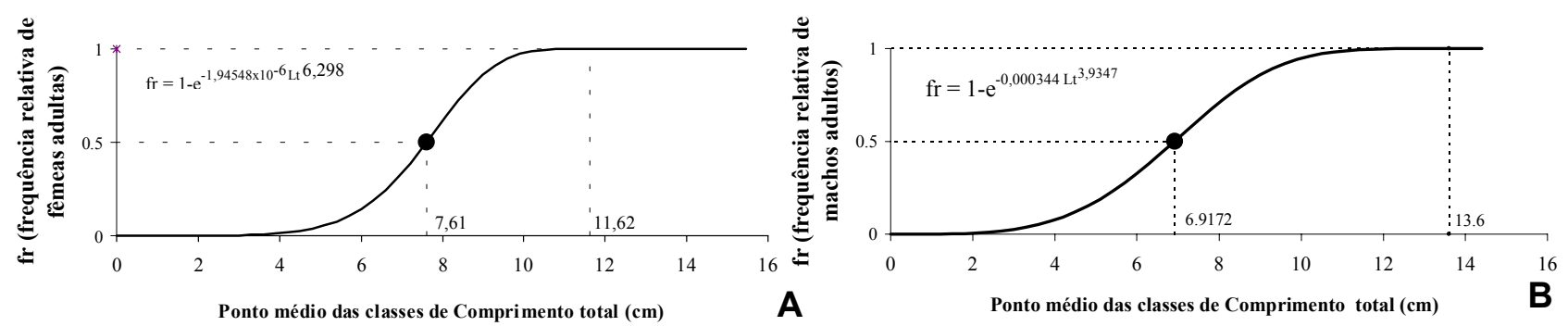

Fig. 7. Curva de tamanho de primeira maturação gonadal para fêmeas (A) e machos (B) de A. brasiliensis.

apenas para os meses outubro (duas fêmeas para cada macho) e dezembro (dois machos para cada fêmea).

A desova de $A$. brasiliensis foi caracterizada sendo do tipo parcelada, comprovada pela observação de ovários semi-desovados, obtidos através da histologia dos ovários. Este resultado coincide com o de Bemvenuti (1987), que trabalhou com a mesma espécie na Lagoa dos Patos.

O maior macho e a maior fêmea coletados apresentaram os comprimentos totais de $14,1 \mathrm{~cm}$ e $15,0 \mathrm{~cm}$ respectivamente, caracterizando a espécie como de pequeno porte.

A curva de primeira maturação foi obtida a partir da transformação linear $Y=6,2982 X-13,15\left(R^{2}=0,9595\right)$ para as fêmeas e $\mathrm{Y}=3,9347 \mathrm{X}-7,9762\left(\mathrm{R}^{2}=0,9729\right)$ para os machos, onde: $\mathrm{Y}=\ln [-\ln (1-\mathrm{fr})]$ e $\mathrm{X}=\operatorname{lnLt}$, sendo as expressões das curvas representadas por:

para fêmeas: $\mathrm{fr}=1-\mathrm{e}^{-1,94548 \times 10^{-6} \mathrm{Lt}^{6,2982}}$

para machos: $\mathrm{fr}=1-\mathrm{e}^{-0,000344 \mathrm{Lt}^{3,9347}}$.

$\mathrm{O}$ tamanho de primeira maturação determinado para fêmeas e machos foi de $7,61 \mathrm{~cm}$ e $6,92 \mathrm{~cm}$ respectivamente (Fig. 7). Bemvenuti (1987), para a mesma espécie na região estuarina da Lagoa dos Patos, obteve comprimentos de primeira maturação gonadal de $10,0 \mathrm{~cm}$ para fêmeas e $9,0 \mathrm{~cm}$ para machos de $A$. brasiliensis, sendo maiores do que os registrados no presente estudo.

\section{AGRADECIMENTO}

Ao Conselho Nacional de Desenvolvimento Científico e Tecnológico (CNPq).

\section{REFERÊNCIAS BIBLIOGRÁFICAS}

Andreata, J.V.; A.M. SaAd; L.A.F. Moraes; C.L. Soares \& A.G. MARCA. 1992. Associações, similaridade e abundância relativa dos peixes da Laguna de Jacarepaguá, Rio de Janeiro, Brasil. Boletim do Museu Nacional, N.S., Zoologia, Rio de Janeiro, 355: 1-25.

Andreata, J.V.; A.G. Marca; C.L. Soares \& R.S. Santos. 1997. Distribuição mensal dos peixes mais representativos da Lagoa Rodrigo de Freitas, Rio de Janeiro, Brasil. Revista Brasileira de Zoologia, Curitiba, 14 (1): 121-134.

Barbieri, L.R.R.; J.V. Andreata; M.A. Santos; M.H.C. Silva; A.S.C. Sebilia \& R.P. Santos. 1991. Distribuição e ciclo de vida das espécies de peixes mais abundantes da Laguna de Marapendi, Rio de Janeiro, Brasil. Revista Brasileira de Zoologia, Rio de Janeiro, 7 (3): 223-243.

Bemvenuti, M.A. 1987. Abundância, distribuição e reprodução de peixes-rei (Atherinidae) na região estuarina da Lagoa dos Patos, RS, Brasil. Atlântica, Rio Grande, 9 (1): 5-32.

Conover, D.O. \& B.E. Kynard. 1984. Field and laboratory observations of spawning periodicity and behavior of a northern population of the Atlantic silverside, Menidia menidia (Pisces: Atherinidae). Environmental Biology of Fishes, Dordrecht, 11 (3): 161-171.

Dias, J. F.; E. Peres-Rios; P.T.C. Chaves \& C.L.D.B. Rossi-WongtsCHowski. 1998. Análise Macroscópica dos Ovários de Teleósteos: Problemas de Classificação e Recomendações de Procedimentos. Revista Brasileira de Biologia, Rio de Janeiro, 58 (1): 55-69.

Figueiredo, J.L. \& N.A. Menezes. 2000. Manual de Peixes Marinhos do Sudeste do Brasil. VI. Teleósteos (5). São Paulo, Museu de Zoologia, Universidade de São Paulo, 116p.

Hostim-Silva, M.; L. Clezar; G.C. Ribeiro \& C. Machado. 1995. Estrutura populacional de Xenomelaniris brasiliensis (Quoy \& Gaimard, 1824) (Osteichthyes - Atherinidae) na Lagoa da Conceição, SC, Brasil. Arquivos de Biologia e Tecnologia, Curitiba, 38 (3): 949-960.

Middaugh, D.P.; G.I. Scott \& J.M. Dean. 1981. Reproductive behavior of the Atlantic silverside, Menidia menidia (Pisces, Atherinidae). Environmental Biology of Fishes, Dordrecht, $6(3 / 4): 269-276$.

Middaugh, D.P. \& M.J. Hemmer. 1992. Reproductive ecology of the inland silverside, Menidia menidia, (Pisces: Atherinidae) from Blackwater Bay, Florida. Copeia, Lawrence, 1: 53-61.

Ortaz, M. \& E. Guerra. 1989. Algunos aspectos de la historia de vida del aterinido Atherinomorus stipes (Pisces: Atherinidae) en una laguna de manglar. Acta Científica Venezolana, Caracas, 40: 280-288.

Palmer, C.J. \& M.B. Culley. 1983. Aspects of the biology of the sandsmelt Atherina boyeri Risso, 1810 (Teleostei: Atherinidae) at Oldbury-upon-Severn, Gloucestershire, England. Estuarine, Coastal and Shelf Science, London, 16: 163-172.

Pessanha, A.L.M.; F.G. Araújo; M.C.C. Azevedo \& I.D. Gomes. 2000. Variações temporais e espaciais na composição e estrutura da comunidade de peixes jovens da Baía de Sepetiba, Rio de Janeiro. Revista Brasileira de Zoologia, Curitiba, 17(1): 251-261.

Pessanha, A.L.M. \& F.G. Araújo. 2001. Recrutamento do peixerei, Atherinella brasiliensis (Quoy \& Gaimard) (Atheriniformes, Atherinopsidae), na margem continental da baía de Sepetiba, Rio de Janeiro, Brasil. Revista Brasileira de Zoologia, Curitiba, 18 (4): 1265-1274.

VAzzolER, A.E. DE M. 1996. Biologia da reprodução de peixes teleósteos: teoria e prática. Maringá, EDUEM, 169p.

Recebido em 18.II.2003; aceito em 21.VIII.2003.

Revista Brasileira de Zoologia 20 (3): 501-506, setembro 2003 